\title{
Acute Organoaxial Gastric Volvulus with cameron lesions - A Dangerous Twist
}

\author{
Justine Chinnappan ${ }^{1}$, Murtaza Hussain $^{1}$, Smit Deliwala ${ }^{1}$, and Ghassan Bachuwa ${ }^{1}$ \\ ${ }^{1}$ Hurley Medical Center
}

February 11, 2022

\begin{abstract}
Acute gastric volvulus is a rare yet life threatening condition with mortality ranging from $30 \%$ to $50 \%$ if not treated promptly. The stomach rotates on itself, causing strangulation precipitating necrosis, cameron lesions and perforation. Long-standing paraesophageal hernia is a common cause of organoaxial gastric volvulus.
\end{abstract}

Acute Organoaxial Gastric Volvulus with cameron lesions - A Dangerous Twist

Justine Chinnapan, MD ${ }^{1}$, Murtaza S. Hussain, MD ${ }^{1}$, Smit S. Deliwala $\mathrm{MD}^{1}$, Ghassan Bachuwa, MD MS FACP MHSA AGSF ${ }^{1}$

${ }^{1}$ Department of Internal Medicine, Michigan State University at Hurley Medical Center, Flint, MI

Correspondence:

Justine Chinnappan, MD

Hurley Medical Center

One Hurley Plaza

Flint, MI - 48503

USA

Key Clinical Message/Abstract:

Acute gastric volvulus is a rare yet life threatening condition with mortality ranging from $30 \%$ to $50 \%$ if not treated promptly. The stomach rotates on itself causing strangulation precipitating necrosis, cameron lesions and perforation. Long-standing paraesophageal hernia is a common cause of organoaxial gastric volvulus.

Key Words: Organoaxial volvulus, cameron lesion, paraesophageal hernia

\section{Case History:}

Acute gastric volvulus is a rare yet life threatening condition with mortality ranging from $30 \%$ to $50 \%$ if not treated promptly. The stomach rotates on itself causing strangulation (Figure 1) precipitating necrosis, cameron lesions and perforation. Long-standing paraesophageal hernia is a common cause of organoaxial gastric volvulus.

Questions: Which complication is an important indication for emergent surgery in long standing Paraesophageal Hernia?

\section{Answer:}


We present a case of paraesophageal hernia complicated with organoaxial volvulus

A 75 year old male with medical history significant for Atrial fibrillation on Warfarin, and longstanding paraesophageal hernia presented with progressive worsening abdominal pain associated with coffee ground emesis of one week duration. Physical exam was remarkable for epigastric tenderness without guarding or rigidity. Laboratory workup revealed Hemoglobin 13.7, PT/INR 72/9.51. Emergent computed tomography angiography demonstrated organoaxial volvulus with the entire stomach in the posterior mediastinum and small duodenal perforation (Figure 2). Gastric decompression with Naso-gastric tube yielded coffee ground fluid. A significant drop in hemoglobin to 7.7 was noted. His PT/INR was reversed with vitamin K and Fresh frozen plasma along with packed Red Blood cell transfusion. Repeat computed tomography illustrated reduction in stomach distention. Esophagogastroduodenoscopy (EGD) revealed a tortuous esophagus with multiple large ulcerations in the body of the stomach and esophagus (Cameron lesions) (Figure 3); with no active bleeding. Patient later underwent laparoscopic transabdominal repair as definitive treatment.

\section{Author Contributions:}

Justine Chinnappan - Acquisition, draft, and review

Murtaza Hussain - Acquisition, draft, and review

Smit S. Deliwala - Conception and review

Ghassan Bachuwa - Review

Conflict of Interest : None to declare

Funding : None to declare

Consent Statement: Published with written consent of the patient.

\section{References:}

Deliwala SS, Hussain MS, Ponnapalli A, Bachuwa G, Gurvits GE. Black oesophagus, upside-down stomach and cameron lesions: cascade effects of a large hiatal hernia. BMJ Case Rep. 2021 Nov 12;14(11):e246496. doi: 10.1136/bcr-2021-246496. PMID: 34772683; PMCID: PMC8593710.

Lopez PP, Megha R. Gastric Volvulus. [Updated 2021 Nov 14]. In: StatPearls [Internet]. Treasure Island (FL): StatPearls Publishing; 2022 Jan-. Available from:https://www.ncbi.nlm.nih.gov/books/NBK507886/
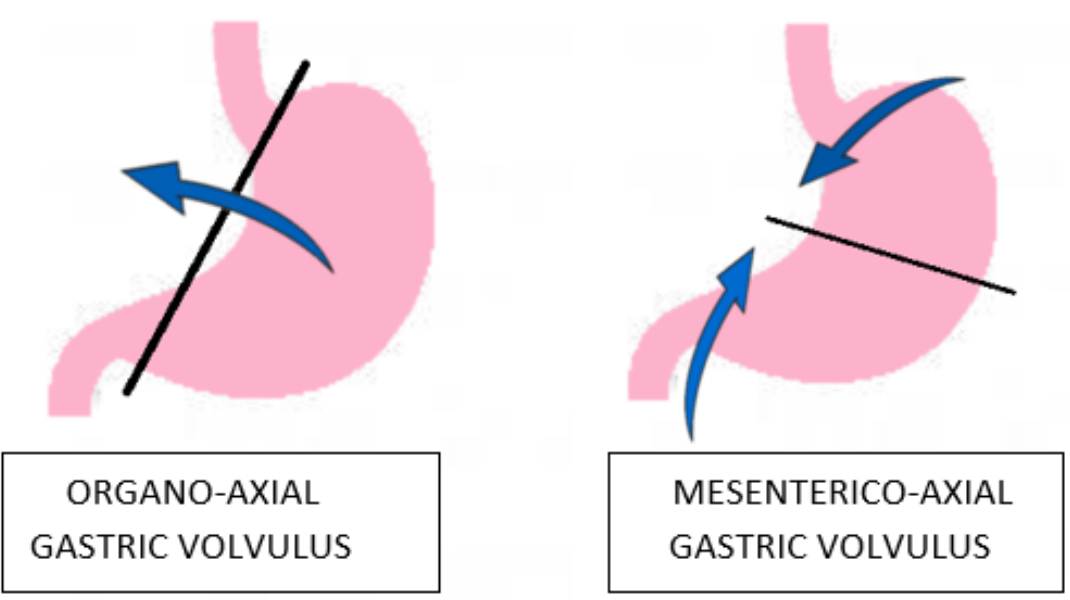


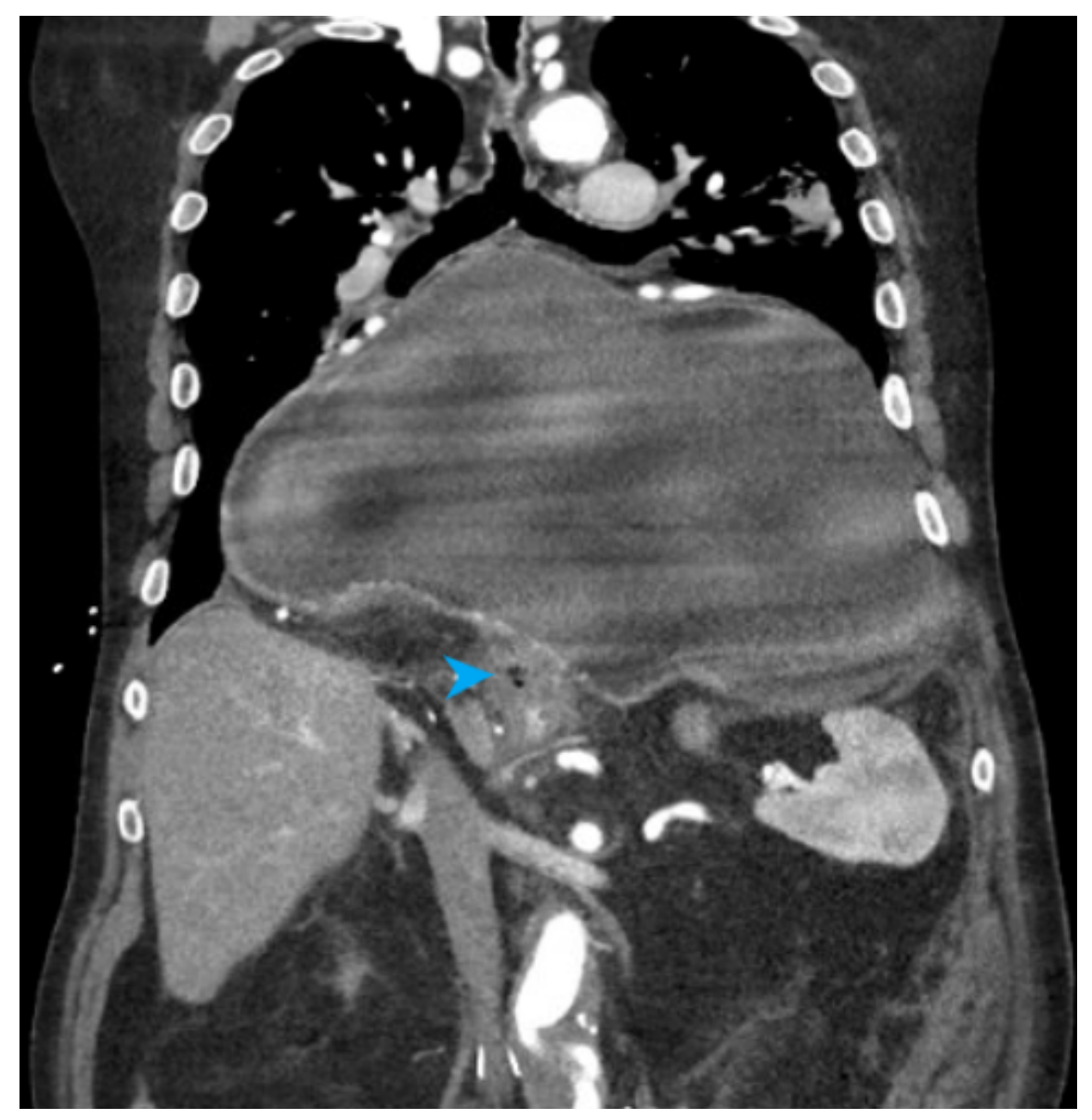




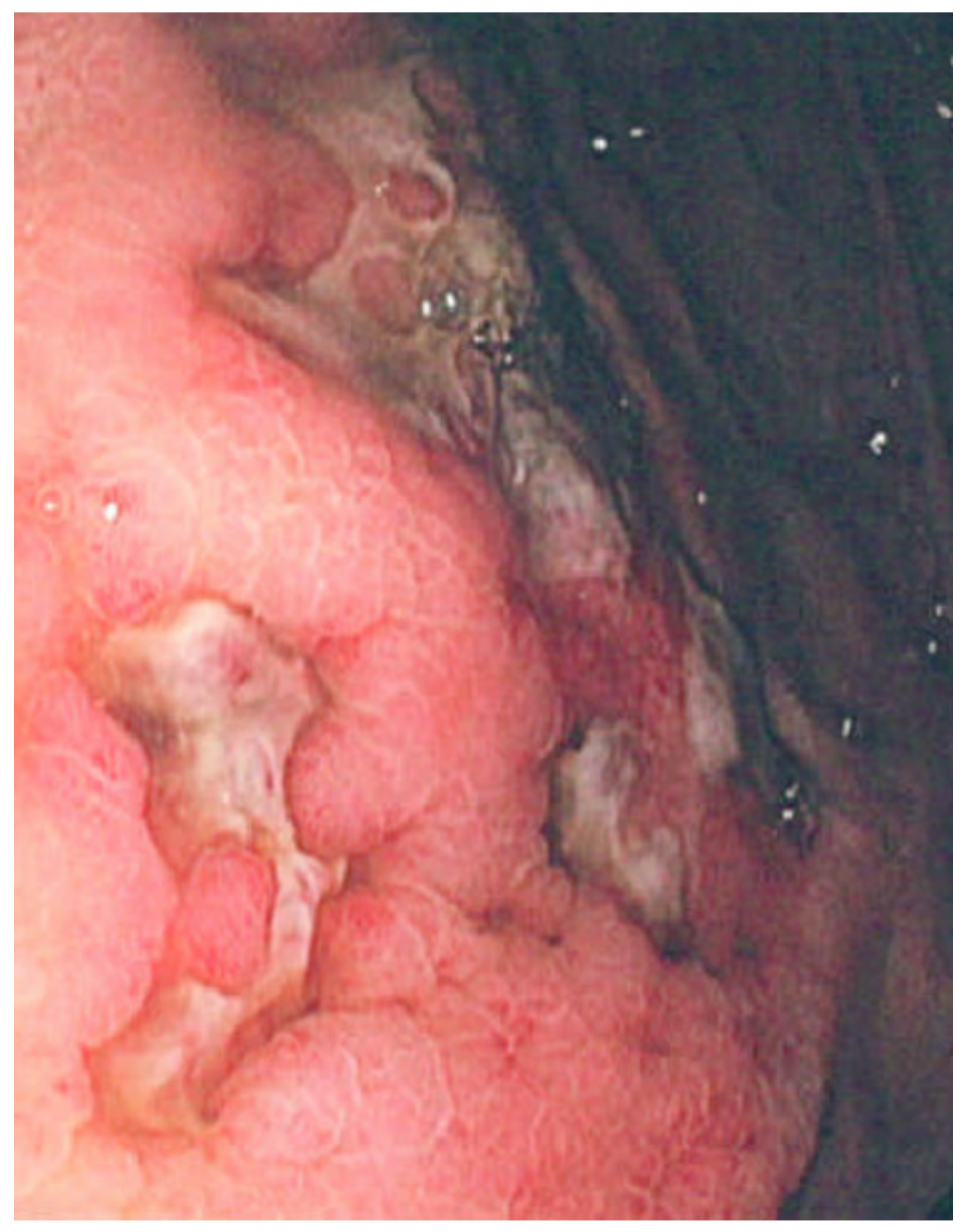

